\title{
Laryngeal Ultrasound in comparison to MDCT and direct laryngoscopy in the assessment of laryngeal carcinoma
}

\author{
Mohamed Salem ${ }^{1}$, Husain Farid weshahy ${ }^{1}$, Moustafa Ezzeldine Radwan ${ }^{2}$, Osama Makhlof ${ }^{3}$, \\ Hossam Galal Elmalah ${ }^{2}$
}

1.ENT Department. Faculty of medicine/Asyut University/Asyut/Egypt.

2. Diagnostic Radiology Department. Faculty of medicine/Asyut University/Asyut/Egypt.

3. ENT Department. Menia general hospital/ Menia/Egypt.

\section{Abstract}

Aim: To evaluate the effectiveness and limitations of ultrasound, in diagnosing laryngeal carcinoma and to compare with Multislice CT and direct laryngoscopy.

Patients and Methods: Thirty patients included in the study were diagnosed with laryngeal carcinoma by clinical examination, direct laryngoscopy, MSCT, High-resolution ultrasound, and endoscopy. To evaluate the visualization ability of ultrasound, CT was regarded as a reference standard.

Results: Thirty male patients, their ages ranged from 45 to 70 years. Hoarseness was the main presenting symptom (80\%). Thirteen patients $(43.3 \%)$ had a glottic lesion. Both laryngoscopy and HRUS showed that 9 patients $(30 \%)$ had good mobility, 15 patients (50\%) had sluggish mobility, 6 patients $(20 \%)$ had no mobility. By MDCT 29 cases (96.7\%) of tumours can be identified, by HRUS 27 cases (90\%) can be identified and 30 cases (100\%) can be identified by direct laryngoscopy. By MSCT from 30 cases (20\%) 6 of the cases showed extra-laryngeal invasion, (76.6\%) 23 of the cases showed no extralaryngeal invasion, and (40\%) 12 cases showed erosion and lyses, (56.7\%) 17 cases showed no erosion and lysis. And by HRUS (10\%) 3 cases showed extra-laryngeal invasion, (83.3\%) 24 of the cases showed no extra-laryngeal invasion, and (30\%) 9 cases showed erosion and lyses, (63.3\%) 18 cases showed no erosion and lysis.There was no statistical difference between MDCT and HRUS in tumour identification, cartilage invasion, deep tumour spread, and cervical lymph node staging.

Conclusion: Ultrasonography is a safe, easy, and effective imaging modality for the diagnosis of laryngeal carcinoma.

Keywords: Laryngeal carcinoma, ultrasonography, MSCT, laryngoscopy

\section{Introduction:}

Imaging and endoscopy are standard techniques in the diagnosis of laryngeal cancer. By employing endoscopy, a tumour emerging from the mucosa can be directly observed. The biopsy can then be done on the same site. It is additionally beneficial in tumour staging because of its capacity to display the laryngeal structure and mobility. ${ }^{1}$
However, determining the depth and extralaryngeal invasion of advanced cancer is challenging, and visualising the inferior section of a bulky lesion that obstructs endoscope transit is sometimes impossible. ${ }^{2}$

The use of computed tomography (CT) in the diagnosis of laryngeal cancer is critical. Dedicated CT scanning can provide important information for tumour staging. 
However, CT cannot be performed in real time and has limitations when assessing laryngeal motility. Ultrasound has the advantage of realtime imaging. It has been developed as a satisfactory imaging modality for diagnosing head and neck diseases. ${ }^{2}$

Sonography is considered the first imaging technique commonly used to detect cervical lymph node metastases from head and neck tumors, but is rarely used as an imaging technique for examining the larynx. ${ }^{4}$

Ultrasonography (US) is appropriate for patients who have a sensitive gag reflex, limited jaw or neck mobility, or stridor. ${ }^{5}$ Laryngeal US is a simple, non-invasive technique for accurately exploring the larynx. ${ }^{6}$ US is generally regarded as a safe imaging modality for pregnancy diagnosis.

Because of the difficulty in visualising laryngeal structures and the acoustic extinction of the ultrasound by the ossified laryngeal cartilages, ultrasound was not widely used in the diagnosis of laryngeal diseases. ${ }^{8}$

The purpose of this study is to compare the efficacy and limitations of Ultrasonography in diagnosing laryngeal carcinoma with Multislice CT and direct laryngoscopy.

\section{Materials and methods:}

\section{Patients:}

Thirty patients included in the study were diagnosed with laryngeal carcinoma in the otolaryngology department, Assiut University Hospital, Assiut, during the period between 2013 and 2016. Ethical clearance for the study was obtained from the institutional Medical Research Ethics Committee. Consent (informed and written) was obtained from each patient.

\section{Inclusion criteria:}

All Patients were recently diagnosed with laryngeal carcinoma.

\section{Exclusion criteria:}

- Patients with non-malignant

laryngeal lesions.

- Patients who had any history of

laryngeal surgery or had

undergone radiotherapy were

excluded prior to surgery.

- Skin fungation of the tumour.

- Patients refusing the research and procedure.

\section{Methods:}

1. History taking, general and ENT examination, including fiber optic laryngoscopy with still photo-documentation.

2. Investigations mainly radiological including MDCT neck and high resolution ultrasound.

3. Endoscopy under general anesthesia and biopsy taking.

\section{CT Larynx}

Multislice CT (MDCT) was performed using (GE Bright speed elite 16 slide, GE Healthcare, USA) in the radiology department of Assiut University.

\section{Data Analysis and Image interpretation:-}

The following data were recorded from each examination:-

1. The exact primary sub-site of the lesion: either Supraglottic, Glottic, subglottic, or transglottic.

2. Nature of lesion (solid, cystic, or mixed solid and cystic).

3. Border of the lesion (well defined or ill-defined).

4. Pattern of enhancement.

5. Deep tumoral extension of the lesions.

6. Cartilage invasion: Depends on four different diagnostic signs in MDCT: (1) extra-laryngeal 
tumor spread, (2) sclerosis, (3) erosion, and (4) lysis.

7. Mucosal surface irregularity.

8. Involvement of supraglottic region (aryepiglottic folds, false vocal cords epiglottis, vallecula.) and glottis region (true vocal cord, anterior and posterior commissures)

9. The subglottic extension of the lesion.

10. Assessment of pyriform sinus and ventricle involvement.

11. Local tumor staging: was based on the criteria for Head and Neck cancer staging according to the TNM scale of the American Joint Committee on Cancer (AJCC 2010) (9).

12. Lymph node involvement, Size, Shape, Density, Pattern of enhancement.

\section{Ultrasound of the larynx:}

High resolution ultrasonography was performed using (Logic P6 GE pro, GE Healthcare, USA) linear probe $10 \mathrm{MHZ}$.

The probe was moved gradually upwards and downwards until the full delineation of laryngeal structures is achieved.

The laryngeal sonographic examination was done during both quiet breathing and phonation to assess the morphology of the lesion and the mobility of the vocal cords respectively.

The hyoid bone was an important landmark that separated the upper airway into two scanning areas: suprahyoid and infrahyoid.

The thyrohyoid membrane connects the hyoid bone's caudal border to the thyroid cartilages cephalad border. It offered a sonographic window through which the epiglottis could be seen.

On the parasagittal and transverse views, the epiglottis was visible as a hypoechoic curvilinear structure through the thyrohyoid membrane.

The tongue protrusion and swallowing aided in the identification of the epiglottis.

On sagittal and parasagittal views, the thyroid cartilage was visible as a linear hypoechoic structure highlighted by the bright A-M interface at its posterior surface. The true and false vocal cords were visible in the transverse view, which had an inverted V shape.

Vocal cord mobility was observed by asking the patient to breath, holding and phonation.

During quiet breathing, the vocal cords normally abduct and adduct to the midline during breath-holding.

Restriction of abduction or adduction movements of the vocal cords indicating the impairment of vocal cord mobility.

Fixation of vocal cords was diagnosed when the vocal cords were in an immobile or fixed state during maneuvers.

The cricoid cartilage appeared round and hypoechoic in the parasagittal view and arch-like in the transverse view.

Extra-laryngeal invasion to the thyroid cartilage, upper cervical lymph nodes, cervical soft tissue, and thyroid gland was also shown.

CT was defined as a reference standard for the competence of sonography due to its wide acceptance and the consideration of surgical and pathological findings as well.

\section{Statistics:}

Data were verified, coded by the researcher, and analyzed using IBMSPSS version 24 (IBM, Chicago, USA).

Means, standard deviations, medians, interquartile ranges (IQR), 
and percentages were calculated as descriptive statistics. The chi-square test was used to compare the differences in the distribution of frequencies between various groups.

ANOVA test was computed to assess the mean differences of data that followed a normal distribution and independent Sample for continuous variables with more than two categories. Statistical significance was defined as a p-value of less than or equal to 0.05 .

\section{Results:}

Thirty male patients their ages ranged from 45 to 70 years with mean age 57.4 years SD \pm 7.91 diagnosed with laryngeal carcinoma. Glottic carcinoma in 13 patients (43.3\%), 6 patients $(20 \%)$ had supraglottic lesion, and 11 patients $(36.7 \%)$ with transglottic lesion

\section{Clinical presentation:}

Hoarseness of voice was the main presenting symptom of all laryngeal lesions 24 cases $(80 \%)$ followed by recurrent attacks of stridor 21 cases (30\%), discomfort sensation and dysphagia 6 cases (20\%), chronic irritative cough 3 cases and aphonia 2 cases (6.7\%). Table (1)

Table (1) Clinical presentation of laryngeal carcinoma

\begin{tabular}{|l|l|}
\hline Symptoms & $\begin{array}{l}\text { Descriptive } \\
\text { statistics (n=30) }\end{array}$ \\
\hline Hoarseness of voice & $24(80 \%)$ \\
\hline Chronic irritative cough & $3(10 \%)$ \\
\hline Stridor & $9(30 \%)$ \\
\hline Dysphagia & $6(20 \%)$ \\
\hline Discomfort sensation & $6(20 \%)$ \\
\hline Hemoptesis & $2(6.7 \%)$ \\
\hline
\end{tabular}

Regarding the assessment of vocal cord mobility by MDCT, HRUS, and fiber optic laryngoscope:

The same result was obtained by HRUS and Direct laryngoscopy except for 2 cases the mobility was not identified by HRUS. So 9 cases
(33.3\%) patients had good mobility, 15 cases $(50 \%)$ had sluggish mobility, 3 cases $(10 \%)$ cases had no mobility and by direct laryngoscopy, 9 cases (30\%) patients had good mobility, 15 cases (50\%) had sluggish mobility, 6 cases (20\%) had no mobility. The correlation between $\mathrm{CT}$ with US and between CT and laryngoscopy were significant $p$ value was less than 0.001 , while it was insignificant between laryngoscopy andlaryngoscopy US.

\section{Regarding Identification of tumours by different modalities:}

By MDCT 29 cases (96.7\%) of tumours can be identified, by HRUS 27 cases $(90 \%)$ can be identified and 30 cases $(100 \%)$ can be identified by direct laryngoscopy. No significant correlation between the three methods of investigations.

\section{Regarding localization of tumors by different modalities:}

By MDCT 29 cases (96.7\%) of tumours can be localized, by HRUS 27 cases $(90 \%)$ can be localized and one case $(3.3 \%)$ cannot be localized by direct laryngoscopy. No significant correlation between the three methods of investigations.

\section{Regarding detection of lymph nodes by different modalities:}

By MDCT 24 cases (80\%) had malignant lymph nodes of central degeneration and loss of hilum, 5 cases $(16.7 \%)$ were have no malignant featuring of lymph nodes

By HDUS 26 cases (86.7\%) were have malignant lymph nodes of central degeneration and loss of hilum, one case $(3.3 \%)$ were have no malignant featuring of lymph nodes with no significant difference.

Final tumor staging according to TNM classification: According to all modalities and confirmation by 
histopathology after biopsy 30 cases are classified as follows. Table (2)

Table (2); staging according to TNM classification

\begin{tabular}{|c|c|c|}
\hline $\begin{array}{l}\text { Tumor } \\
\text { staging }\end{array}$ & Number & \% \\
\hline Stage 0 & 0 & $0 \%$ \\
\hline Stage 1 & 8 & $26.7 \%$ \\
\hline Stage 2 & 6 & $20 \%$ \\
\hline Stage 3 & 15 & $50 \%$ \\
\hline Stage 4 & 1 & $3.3 \%$ \\
\hline
\end{tabular}

\section{Assessment of cartilage invasion:}

By MSCT from 30 cases (20\%) 6 cases showed extra-laryngeal invasion, $(76.6 \%) 23$ of cases shows no extralaryngeal invasion and (40\%) 12 cases showed erosion and lyses, (56.7\%) 17 cases showed no erosion and lysis.

And by HRUS (10\%) 3 cases showed extra-laryngeal invasion, $(83.3 \%) 24$ cases shows no extralaryngeal invasion and (30\%) 9 cases showed Erosion and lyses, (63.3\%) 18 cases shows no erosion and lysis. Table (3)

Table (3): Cartilage invasion

\begin{tabular}{||l|c||c||c||}
\hline Laryngeal cartilage invasion & MDCT & HRUS & P value \\
\hline \hline Extralaryngeal & & & \\
No & $23(76.7 \%)$ & $24(80 \%)$ & 0.364 \\
Yes & $6(20 \%)$ & $3(10 \%)$ & \\
Not identified & $1(3.3 \%)$ & $3(10 \%)$ & \\
\hline \hline Erosion and lysis & & & \\
No & $17(56.7 \%)$ & $18(60 \%)$ & 0.483 \\
Yes & $12(40 \%)$ & $9(30 \%)$ & \\
Not identified & $1(3.3 \%)$ & $3(10 \%)$ & \\
\hline
\end{tabular}

Table (4): deep tumoral extension

\begin{tabular}{|c|c|c|c|}
\hline Deep tumoral extension & MDCT & HRUS & P value \\
\hline $\begin{array}{l}\text { Paraglottic } \\
\text { No } \\
\text { Yes } \\
\text { Not identified }\end{array}$ & $\begin{array}{c}17(56.7 \%) \\
12(40 \%) \\
1(3.3 \%)\end{array}$ & $\begin{array}{c}19(63.3 \%) \\
9(30 \%) \\
2(6.7 \%)\end{array}$ & 0.646 \\
\hline $\begin{array}{l}\text { Pre-epiglottic } \\
\text { No } \\
\text { Yes } \\
\text { Not identified }\end{array}$ & $\begin{array}{c}14(46.7 \%) \\
15(50 \%) \\
1(3.3 \%)\end{array}$ & $\begin{array}{c}16(53.3 \%) \\
12(40 \%) \\
2(6.7 \%)\end{array}$ & 0.670 \\
\hline $\begin{array}{l}\text { Extralaryngeal } \\
\text { No } \\
\text { Yes } \\
\text { Not identified }\end{array}$ & $\begin{array}{c}23(76.7 \%) \\
6(20 \%) \\
1(3.3 \%)\end{array}$ & $\begin{array}{c}25(83.3 \%) \\
3(10 \%) \\
2(6.7 \%)\end{array}$ & 0.493 \\
\hline $\begin{array}{l}\text { Cartilage invasion } \\
\text { No } \\
\text { Yes } \\
\text { Not identified }\end{array}$ & $\begin{array}{c}20(66.7 \%) \\
9(30 \%) \\
1(3.3 \%)\end{array}$ & $\begin{array}{c}22(73.3 \%) \\
6(20 \%) \\
2(6.7 \%)\end{array}$ & 0.598 \\
\hline $\begin{array}{l}\text { Vessel invasion } \\
\text { No } \\
\text { Yes } \\
\text { Not identified }\end{array}$ & $\begin{array}{c}30(100 \%) \\
0(0 \%) \\
0(0 \%)\end{array}$ & $\begin{array}{c}30(100 \%) \\
0(0 \%) \\
0(0 \%)\end{array}$ & \\
\hline
\end{tabular}




\section{.Case 1: Right glottic carcinoma}
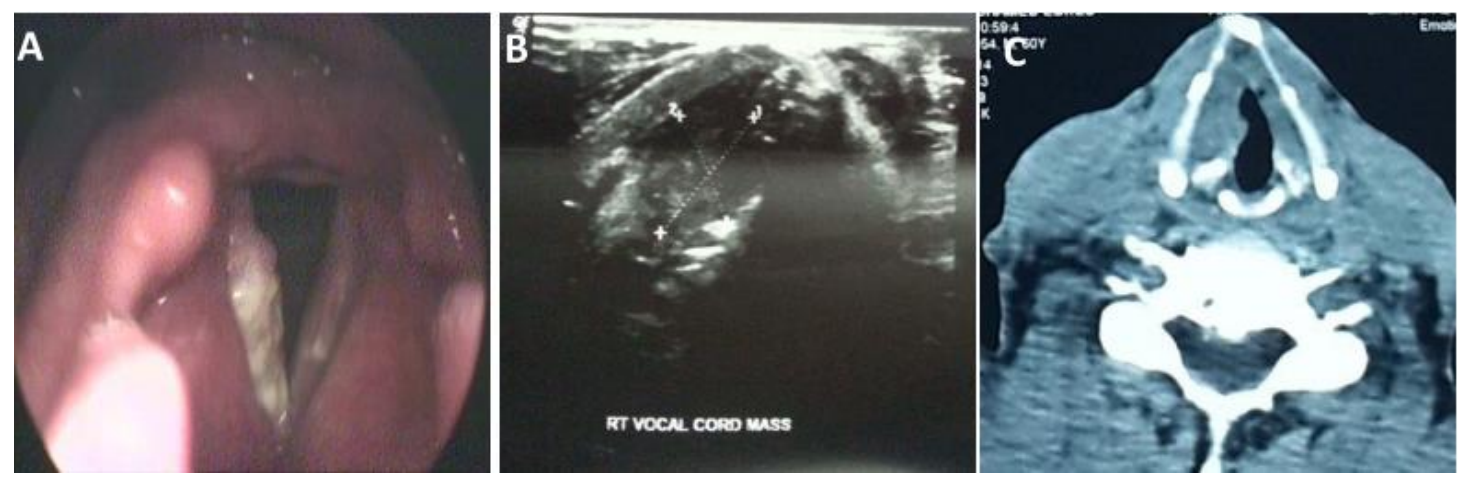

Figure 1:- (a) fiber optic laryngoscopy picture showing soft tissue swelling arising from the anterior third of the right vocal cord, with mucosal surface irregularity. (b) Cross-sectional ultrasound images showing well-defined hypoechoic lesion seen centered upon the right vocal cord and reaching the anterior commissure exceeding $2 \mathrm{~cm}$. (c) axial CT image showing hypodense lesion seen at the right vocal cord reaching the anterior commissure with obliteration of the related fat plans (the paraglottic space) and slight encroachment upon the related air column

\section{Case 2: Right supraglottic carcinoma}
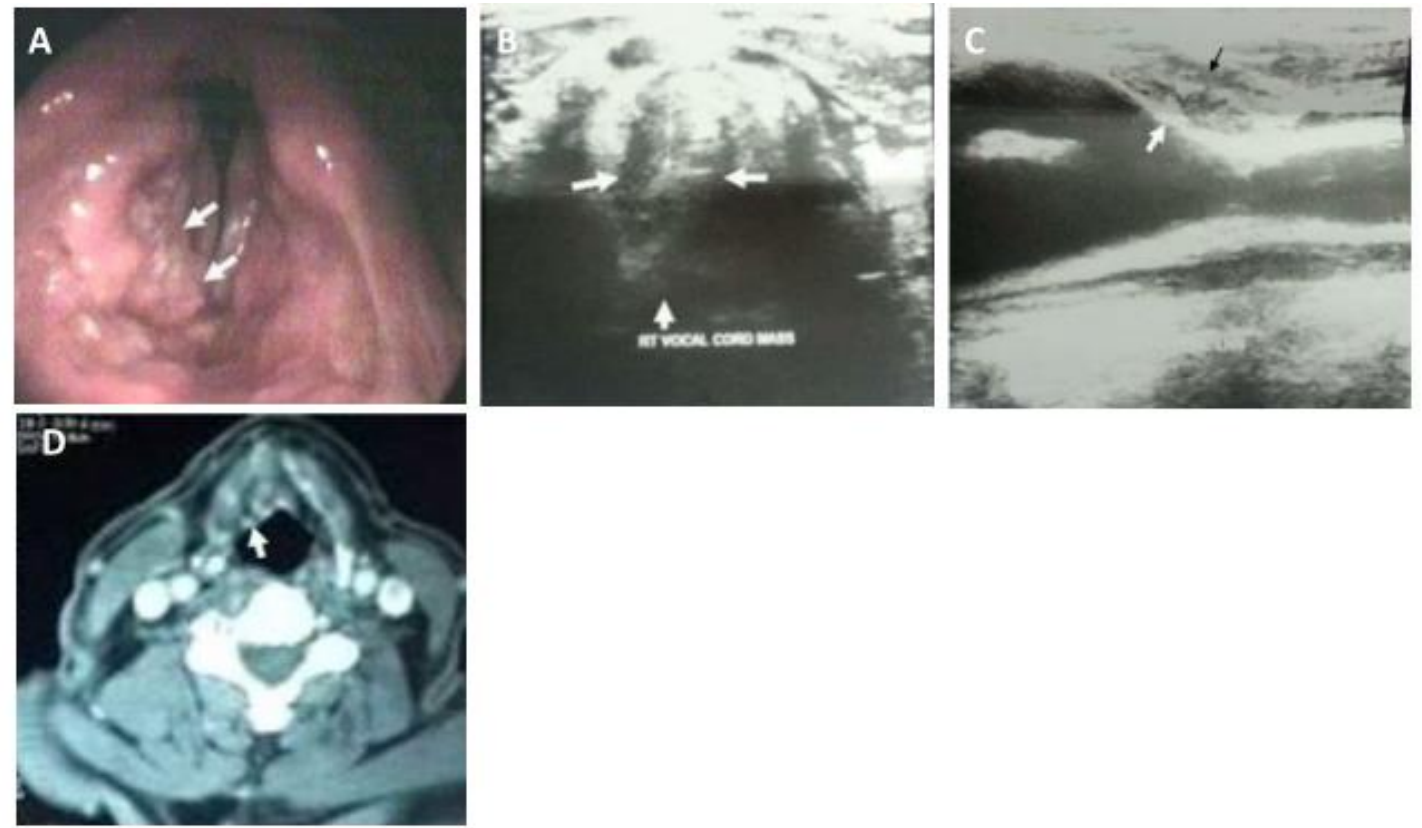

Figure 2: (a) fiber optic laryngoscopy picture showing right supraglottic swelling seen infiltrating the tongue base, valleculae, epiglottis, and right aryepiglottic fold. (b) Ultrasound images showing welldefined hypoechoic lesion seen in the right supraglottic region reaching the tongue base. (c) Ultrasound images showing enlarged regional lymph node with distorted architecture. (d) Axial CT image showing hypodense lesion seen supraglottic with an irregular surface. (The pre-epiglottic space). 


\section{Assessment of deep tumoral extension:}

By MDCT, $12(40 \%)$ cases showed paraglottic space extension, $15(50 \%)$ cases showed pre-epiglottic space extension.

And by HRUS, 9 (30\%) cases showed paraglottic space extension, 12 (40 \%) cases showed pre-epiglottic space extension Table (4).

\section{Discussion:}

The criteria standard for evaluating laryngotracheobronchial pathologic disorders is still direct examination by endoscopy. Mucosal and superficial submucosal lesions can be easily visualised and diagnosed using endoscopy. Deeper structures, on the other hand, can only be evaluated via CT scanning or MRI. For general laryngeal imaging, CT scanning has become the most popular method. It can be found in most hospitals and even some outpatient clinics. The acquisition period for a CT scan is incredibly fast (less than a second), which is ideal for laryngeal examinations because patients are usually asked to hold their breath to reduce movement. ${ }^{10,11}$

Ultrasonography is routinely done for patients with head and neck tumors to detect cervical lymph nodal metastases, yet so far it is not routinely used for diagnosis and staging laryngeal neoplasm. ${ }^{12}$

This is owing to the idea that in adults, the soft-tissue-air interface and calcified thyroid cartilage prevent laryngeal structures from being seen. Despite the varying stages of thyroid cartilage calcification, most laryngeal structures can be examined using ultrasound. The thyrohyoid and cricothyroid membranes are good places for the ultrasound beam to go. ${ }^{13}$

Our study aimed to evaluate the efficiency and limitations of ultrasonography, in diagnosing different laryngeal carcinomas, 30 patients were included in this study.

HRUS was very accurate in diagnosing glottic (43.3\%), supraglottic (20\%), transglottic $(36.7 \%)$ carcinoma, which is the same result obtained from MDCT which is a similar result reported by the study of Khalil et al., (2011) ${ }^{14}$ except 3 cases not diagnosed. One patient was diagnosed histopathologically to have CIS (carcinoma in situ) which appeared as a white patch on the top surface of the vocal folds about $2 \mathrm{~cm}$ in diameter, this patch was found difficult to be detected by HRUS which agrees with Shigehiko et al., (2009). ${ }^{15}$

Two cases were diagnosed laryngoscopically and by MSCT as transglottic carcinoma and were tracheostomized and cannot be detected by HRUS due to air inflation only malignant lymph node were seen and detected. According to the study of $\mathrm{Hu}$ et al. (2012) ${ }^{16}$, ultrasonography correctly identified the majority of laryngeal carcinomas (35 of 36, or 97.2 percent), with only one T2 stage aryepiglottic fold tumour overlooked. They discovered that both ultrasonography and contrastedenhanced CT showed a high level of pretherapeutic staging accuracy for laryngeal cancer, with ultrasonography scoring 83.3 percent and CT scoring $88.8 \%$ (p 140.735$)$. 
Regarding the mobility, the same result was obtained by HRUS and Direct laryngoscopy so (30\%) patients had good mobility, (50\%) had sluggish mobility, $(20 \%)$ cases had no mobility and the mobility were not identified by MDCT which matched the result of Raghavendra et al., (1994). ${ }^{17}$

As regards lymph node detection by MDCT $24(80 \%)$ of cases had malignant lymph nodes of central degeneration and loss of hilum and by HRUS $26(86.7 \%)$ of cases were have no malignant featuring of lymph nodes which is comparable to Khalil et al., (2011). ${ }^{14}$ Beale et al. (2020) hypothesised that prelaryngeal nodes (Delphian nodes), a major route of lymphatic spread in laryngeal cancer, can be identified more easily with US than with CT or MRI, where distinguishing from the strap muscles may be problematic. ${ }^{13}$

The presence of cartilage invasion is crucial for laryngeal carcinoma staging and outcome. The presence of neoplastic cartilage invasion not only limits voice-saving partial laryngectomy, but it also alters the response to radiation therapy, increasing the risk of tumour recurrence. ${ }^{18}$

According to the literature, US has a high sensitivity, but even more crucially, a high specificity for the assessment of cartilaginous tumoral invasion (sensitivity: 66.6\%-87.5\%; specificity: $92.9 \%-97.9 \%) .{ }^{12,19}$

In our study by MSCT of 30 cases, 6 the cases $(20 \%)$ showed extralaryngeal invasion, $23(76.6 \%)$ of cases shows no extralaryngeal invasion and 12 $(40 \%)$ of the cases showed erosion and lyses, $17(56.7 \%)$ cases showed no erosion and lysis. By HRUS 3 the cases $(10 \%)$ showed extralaryngeal invasion, $25(83.3 \%)$ the cases show no extralaryngeal invasion and $9(30 \%)$ the cases showed erosion and lyses, 19
(63.3\%) cases shows no erosion and lysis which is the same result obtained from Erkan et al., (1993) ${ }^{18}$ except 2 cases $(6.7 \%)$ not identified by HRUS.

Erkan et al. (1993) reported 100\% sensitivity for detecting laryngeal cartilage invasion by sonography.

Our findings were in line with those of $\mathrm{Hu}$ et al. (2012) ${ }^{16}$, who found no significant differences in the identification of cartilage invasion between ultrasonography and contrastenhanced CT.

Sonography and contrast-enhanced CT had sensitivity, specificity, and accuracy of $88.6,85.5$, and $86.4 \%$, and $82.9,91.6$, and $89 \%$, respectively. Also in our study by MDCT, 12 (40\%) cases showed paraglottic space extension, $15(50 \%)$ cases showed pre-epiglottic space extension, and 6 $(20 \%)$ cases showed extralaryngeal extension, and 9 (30\%)cases were showed cartilage invasion. While, by HRUS, $9(30 \%)$ cases showed paraglottic space extension, $12(40 \%)$ cases showed pre-epiglottic space extension, and $3(10 \%)$ cases showed extralaryngeal extension, and $6(20 \%)$ cases were showed cartilage invasion. Gritzmann et al., (1989) ${ }^{20}$ reported a similar result. According to $\mathrm{Hu}$ et al. (2012) ${ }^{16}$, ultrasonography was correct in $86.1,94.4,83.3$, and $97.2 \%$ of cases for the invasion of the paraglottic space, pre-epiglottic space, subglottic region, and extralaryngeal tissues, and CT was correct in $91.7,94.4,88.9$, and $100 \%$ of cases for the invasion of the paraglottic space, pre-epiglottic space, subglottic region, and Sonography and CT had no statistically significant differences.

Laryngeal US is an easily reproducible and non-invasive technique that can accurately explore the larynx. ${ }^{6}$ High resolution ultrasound has several advantages over current methods, including its noninvasive 
nature, absence of ionizing radiation, and its ease of use in the ambulatory setting. ${ }^{21}$

Because of substantial technological advancements, portable ultrasound equipment are now more accessible, inexpensive, and of great diagnostic value. These advancements now provide otolaryngologists with a portable instrument that improves diagnostic accuracy, simplifies common procedures, and improves patient convenience and safety. ${ }^{22}$

The ultrasound appearance of the laryngeal region has long been a clinical problem, thus a thorough understanding of the head and neck complex was required. Any abnormality can be easily identified with a good understanding of the typical radiological anatomy of the head and neck. Ultrasonography imaging has grown in popularity as a strong diagnostic technique, particularly for examining the head and neck. Ultrasound technology has advanced, resulting in higher picture quality and tissue distinction, while also being non-time consuming and repeatable, with the added benefit of dynamic examination of the voice cords. ${ }^{23}$

The capacity to perform targeted biopsies in places that would otherwise be inaccessible is a diagnostic advantage of US over cross-sectional imaging. This is especially true for patients who cannot have general anaesthesia, for those who have clinical suspicion of submucosal recurrence, and for those who have had a previous negative biopsy. A biopsy of the thyrohyoid membrane is usually done transcutaneously. When there are small focused areas that are suspicious for submucosal recurrence, intraoperative localization and confirmation of resection may be undertaken. ${ }^{13}$
Laryngeal ultrasonography is very reliable in uncooperative patients having aggressive gag reflex or those suffering any disease interfering with the introduction of endoscopes. It can be a non-invasive complementary technique for pretherapeutic staging of laryngeal carcinoma, but still not able to detect masses less than $2 \mathrm{~cm}$ and masses present in trachostomized patients which are under study.

Finally, we recommend training courses for the radiologists who are more concerned about researches on HRUS on the larynx.

\section{Conclusion:}

This study demonestrated that laryngeal US is as effective as CT in evaluation of laryngeal carcinoma and each of them complement the armamentorium of investigation for those patients.

\section{References:}

1-Mandell DI. Head and neck anomalies related to branchial apparatus. Otolaryngology clinic north American. 2000, 33(6) 1309-1032.

Graham A. The development and evaluation of the pharyngeal arches. J ant. 2001, 199(pt.1-2), 133-141.

3-Manoukian JJ., Tan AK. Embryology of the larynx . In: Tewfik TL, Der Kaloustian VM, eds. Congenital Anomalies of The Ear Nose, and Throat. New York,NY :Oxford University Press. 1997, 377-382.

4-Moore KL, Persaud TVN. The Developing Human Clinically Orientated Embryology 6thedition .Philadelphia W.B. Saunders. 1998

5-Hartnick CJ and zeitels SM. Pediatric video laryngostroboscopy. Int $\mathrm{j}$ pediatr otorinolaryngol. 2005, 69:215-219.

6-Strauss S. Sonographic appearance of cricoid cartilage calcification in 
healthy children. AJR Am J Roentgenol. 2000, 174:223-228.

7-Wendy D. Laryngeal Ultrasound provides noninvasive assessment of vocal fold lesions.Annals of Otology Rhinology,and Laryngology. 2007, 171:631-647

8-Arens C and Glans H. Endoscopic high frequency ultrasound of the larynx. Eur.Arch.Otolaryngol,. 2003, 256:316-322.

9- The American Joint Committee on Cancer: the 7th edition of the AJCC cancer staging manual and the future of TNM. Ann Surg Oncol. 2010 Jun;17(6):1471-4.

10-Chang EW,Kobler JB, Yun SH. Triggered opticalcoherence tomography for capturing rapid periodic motion, Sci Rep. 2011,1:48

11-Stratmann S, Huang JC, Maki K, Hatcher D, Miller AJ.Three dimensional analysis of the airway with cone-beam computed tomography. Am J Orthod Dentofacial Orthop.2011,104(5):607-15

12-Xia CX, Zhu Q, Zhao HX, Yan F, Li SL, Zhang SM. Usefulness of ultrasonography in assessment of laryngeal carcinoma. $\mathrm{Br} \mathrm{J}$ Radiol 2013;86(1030):20130343

13-Timothy Beale, Victoria M. Twigg ,Mariana Horta ,Simon Morley. HighResolution Laryngeal US: Imaging Technique, Normal Anatomy, and Spectrum of Disease.RadioGraphics 2020; 40:775-790 .

14-Khalil T , Madyan Y.Farid A. High resolusion ultrasound for diagnosis of vocal cord lesions; Ejentas ,December 2011, 11, 64- 68.

15-Shigehiko kuribayashi, Tsuguhiro Miyashita , Munenaga Nakamizo, Shinichiro Kumita. Utility of Sonography for evaluation of clinical T1 and T2 Glottic Carcinoma.; J Ultrasound Med . 2009,281429-1440
16-Hu Q, Luo F, Zhu SY, Zhang Z, Mao YP, Hui Guan X. Staging of laryngeal carcinoma: comparison of highfrequency sonography and contrast-enhanced computed tomography. Clin Radiol 2012;67(2):140-147.

17-Raghavendra BN., Horri SC., Reede DL.,Romancik WM, Presky M, Bergeron RT. Sonographic anatomy of the larynx with particular reference to vocal cords.J Ultrasound.Med. 1994,6:225-230.

18-ERKAN M, Tolu I, Aslan T et al. Ultrasonography in laryngeal cancers . J laryngol otol. 1993, 107:65-8.

19-Hu Q, Zhu SY, Zhang Z, Luo F, Mao YP, Guan XH. Assessment of glottic squamous cell carcinoma: comparison of sonography and noncontrast-enhanced magnetic resonance imaging. J Ultrasound Med 2011;30(11):1467-1474

20-Gritzmann N, Traxler M, Grasl M. Pavelka R. Advanced laryngeal cancer: sonographic assessment. Radiology. 1989, 171:171-175.

21-Paul C. Bryson, MD; W. Derek Leight, MD. Carlton J. Zdanski, MD; Amelia F. Drake, MD; Austin S. Rose, MD High-Resolution Ultrasound in the Evaluation of Pediatric Recurrent Respiratory Papillomatosis Arch Otolaryngol Head neck surg. 2009, vol 135 ( 3), 250- 253.

22- Ol'khova E, IuL S, Onufrieva E, Shchepin N Laryngeal ultrasound studies: potential, prospects, limitations. Vestn Otorinolaringol. 2009, 5:9-12

23-ALEEM M, NASSER HM. Ultrasound diagnostic value in assessment of vocal cord mobility before neck surgery. Med J Cairo Univ. 2019, 87(December): 4371-4377 